\title{
ASERTIVIDAD: UNA HABILIDAD SOCIAL NECESARIA EN LOS PROFESIONALES DE ENFERMERÍAY FISIOTERAPIA
}

\author{
Wilson Cañón-Montañez $z^{1}$ Alba Luz Rodríguez-Acelas ${ }^{2}$
}

\footnotetext{
${ }^{1}$ Mestre en Epidemiología. Profesor del Programa de Enfermería de la Facultad de Salud de la Universidad de Santander. Bucaramanga, Colombia. E-mail: wcanon@udes.edu.co

${ }^{2}$ Mestre en Enfermería. Profesor del Programa de Enfermería de la Universidad Cooperativa de Colombia, Bucaramanga, Colombia. E-mail: alra1900@yahoo.com
}

RESUMEN: Los profesionales de la salud están constantemente interrelacionados con otras personas, en algunas ocasiones de éstas relaciones se derivan situaciones angustiantes y conflictivas. El objetivo del presente estudio es conocer la asertividad de estudiantes universitarios de enfermería y fisioterapia. Es un estudio de corte transversal. Se incluyeron 225 estudiantes. El instrumento empleado fue el Inventario de Asertividad de Rathus. Los resultados muestran que la edad es un elemento fundamental para que las personas sean asertivas; a medida que aumenta la edad, aumenta la asertividad indirecta por cada año en (0.04) y disminuye la no asertividad $(p=0.004)$. Lo anterior, permite concluir que la asertividad como habilidad relacional debe ser contemplada como requisito en la formación de los profesionales de enfermería y fisioterapia, dado que aplicada correctamente permite una mayor y más completa consecución de los cuidados y servicios que se prestan, lo que proporciona reconocimiento profesional y personal.

DESCRIPTORES: Relaciones interpersonales. Asertividad. Análisis factorial. Profesionales de salud. Educación superior.

\section{ASSERTIVENESS: A NECESSARY SOCIAL ABILITY IN THE PROFESSIONALS OF NURSING AND PHYSIOTHERAPY}

\begin{abstract}
Health professionals are constantly interrelated with other people, sometimes derived from these relationships and conflicting distressing situations. The aim of this study was to determine the assertiveness of college students in nursing and physiotherapy. It is a cross-sectional study. 225 students were included. The instrument used was the Rathus Assertiveness Schedule. The results show that age is an essential element for people to be assertive; with increasing age, the indirect assertiveness increases each year in $(0.04)$ and decreases the non-assertiveness $(\mathrm{p}=0.004)$. This leads to the conclusion that assertiveness as ability relational should be seen as a requirement in the formation of the professionals of nursing and physiotherapy, as properly applied enables greater and more complete realization of care and services provided, providing professional and personal recognition.
\end{abstract}

DESCRIPTORS: Interpersonal relations. Assertiveness. Factor analysis. Health professionals. Higher education.

\section{ASSERTIVIDADE: UMA CAPACIDADE SOCIAL NECESSÁRIA NOS PROFISSIONAIS DE ENFERMAGEM E FISIOTERAPIA}

RESUMO: Os profissionais da saúde estão constantemente envolvidos com outras pessoas e algumas vezes estas relações produzem situações conflitivas e angustiantes. O objetivo deste estudo foi determinar a assertividade dos acadêmicos de enfermagem e fisioterapia. É um estudo transversal. 225 estudantes foram incluídos. O instrumento utilizado foi o Inventário de Assertividade Rathus. Os resultados mostram que a idade é um elemento essencial para que as pessoas sejam assertivas, com o aumento da idade, aumenta a assertividade indireta em cada ano $(0,04)$ e diminui a não-assertividade $(\mathrm{p}=0.004)$. Isso leva à conclusão de que as competências de assertividade relacional deve ser visto como uma exigência na formação do profissional de enfermagem e fisioterapia, cuja correta aplicação permite uma realização maior e mais completa dos cuidados e dos serviços prestados, proporcionando reconhecimento profissional e pessoal. DESCRITORES: Relações interpessoais. Assertividade. Análise fatorial. Profissionais da saúde. Ensino superior. 


\section{INTRODUCCIÓN}

En cuanto seres sociales, las personas están la mayor parte de su tiempo interactuando con los demás; no es ningún secreto que uno de los aspectos que más aprecian de su existencia, es precisamente el de las relaciones sociales. De hecho, el poseer buenas habilidades sociales determina en gran medida (facilitando o entorpeciendo) la búsqueda de la felicidad; sin embargo conseguir que las relaciones interpersonales, la comunicación y el diálogo con el entorno sean naturales, espontáneos, claros, sin malentendidos ni conflictos, es más difícil de lo que a primera vista pudiera parecer; la dificultad e importancia del fenómeno radica, en que las relaciones con otros pueden ser el origen de las mejores satisfacciones o los mayores conflictos. De esta manera, mientras que muchos encuentros cotidianos pueden ser desagradables, debido a un comportamiento social inadecuado, otros, cuando son bien llevados producen alegría por la vida. ${ }^{1}$

Ahondando al respecto, en las ciencias de la salud, se pone mucho énfasis en la adquisición de competencias técnico-científicas, más que en la adquisición de habilidades sociales e interpersonales. Los profesionales de la salud están constantemente interrelacionados con otras personas, en algunas ocasiones de éstas relaciones se derivan situaciones angustiantes y conflictivas, que son potentes generadores de estrés y pueden dificultar el buen desempeño del rol profesional; es por esto, que en muchas ocasiones la gente se corta al hablar, no sabe pedir un favor, le cuesta ir sola a realizar actividades sencillas, no puede comunicar lo que siente, no sabe resolver situaciones con los amigos o con la familia, o tal vez carezca de amistades, entre otras situaciones. En todas estas dificultades se observa la falta de habilidades sociales. Un estudioso en el año 2007 afirmó que "la conducta socialmente habilidosa es ese conjunto de comportamientos emitidos por un individuo en un contexto interpersonal que expresa los sentimientos, actitudes, deseos, opiniones o derechos de ese individuo de un modo adecuado a la situación, respetando esas conductas en los demás y que generalmente resuelve los problemas inmediatos de la situación mientras minimiza la probabilidad de futuros problemas". 2:6

Ahora bien, aunque es en la infancia cuando se aprende en mayor medida a relacionarse con los demás, este proceso continua durante toda la vida; es muy probable que en alguna ocasión cualquier individuo haya tenido dificultad en defender sus derechos, expresar sentimientos $\mathrm{u}$ opiniones, defender el propio punto de vista sin agredir a los demás, iniciar nuevas relaciones o simplemente poner punto final a una conversación. Es aquí, donde la asertividad funciona como una habilidad social, pero ¿que es la asertividad? La asertividad fue descrita inicialmente en 1949 como "un rasgo de personalidad"3:21, se pensó que algunas personas la poseían y otras no; más tarde otro experto en 1958 entendía la asertividad como "la expresión adecuada, hacia otra persona, de cualquier emoción que no sea respuesta de ansiedad"4:76 y en 1966 otro autor la definió como la "expresión de los derechos y sentimientos personales" . 5:209 Por su parte, otros en 2003 consideraron que la asertividad es la conducta que permite a una persona actuar para implantar su propio interés, defenderse a sí mismo sin ansiedad y expresar sus derechos, sin destruir los derechos de otros. ${ }^{1}$

Históricamente, las definiciones de la asertividad siguen manteniendo los mismos principios, según otro investigador en 1999 “asertividad significa defender y hablar por uno mismo sin lesionar los derechos de los demás", 6:368 mientras que para otro autor en 2010 mantiene una definición clásica en donde la asertividad es la capacidad de autoafirmar los propios derechos, sin dejarse manipular y sin manipular a los demás. ${ }^{7}$ Finalmente resalta analizar la asertividad donde su mensaje básico es: "esto es lo que pienso, esto es lo que yo siento, ésta es la forma en la que yo veo la situación" 8:211 y se dice sin dominar, humillar o degradar al otro individuo. En este sentido, se podrían retomar tres elementos generales de las definiciones: uno, es que el individuo tiene derecho de expresarse; dos, es necesario también el respeto hacia el otro individuo y tres, es deseable que las consecuencias del comportamiento sean satisfactorias para ambos miembros de la interacción ${ }^{1}$.

Ser un profesional asertivo permitirá obtener beneficios tanto en el área personal, aumentando su autoestima, su seguridad personal, su autoimagen y su autoconcepto, como en el área laboral, disminuyendo el número de situaciones estresantes e incrementando la eficacia, calidad y competencia de los cuidados y servicios que se prestan. A pesar de ello, tanto la asertividad como el manejo de las técnicas de habilidades sociales son poco conocidas para la mayoría de los profesionales de enfermería. ${ }^{9}$

Dada la importancia de la asertividad en el campo de la salud, se planteó el presente estudio, con el objetivo de conocer la asertividad de los 
estudiantes de Enfermería y Fisioterapia de la Universidad Industrial de Santander de Bucaramanga-Colombia.

\section{MÉTODOS}

\section{Diseño}

Estudio observacional de corte transversal. ${ }^{10}$

\section{Población y muestra}

El total de estudiantes fue de 404, de los cuales 221 pertenecían a la Escuela de enfermería y 183 a la Escuela de fisioterapia de la Universidad Industrial de Santander, Ciudad de BucaramangaColombia, matriculados en el segundo periodo académico del 2003. La población de estudio estuvo constituida por 225 estudiantes, 174 pertenecían a la Escuela de Enfermería y 51 a la Escuela de Fisioterapia. El tipo de muestreo utilizado fue no probabilístico intencional o de conveniencia. Los investigadores seleccionaron directa e intencionadamente a la población de estudio con quienes se tenía una relación académica.

\section{Instrumento}

Para la recolección de la información se utilizó el Inventario de Asertividad de Rathus (1973) "Assertiveness Schedule Rathus" (RAS). ${ }^{11}$ El RAS tiene una consistencia interna de 0.79. Éste es un cuestionario que consta de 30 reactivos y evalúa la capacidad del individuo para hacer valer sus derechos ante situaciones de consumo o de servicio, con figuras externas o de negocios, iniciación social y sentimientos hacia otros, con una escala tipo likert con seis probabilidades de respuesta $(+3$ muy característico de mí, +2 bastante característico de mí, +1 algo característico de mí, -1 algo no característico de mí, -2 bastante poco característico de mí y -3 muy poco característico de mí), con el cual se pretendía evaluar la asertividad de los sujetos de estudio. El resultado de la puntuación final podía oscilar entre -90 (mínima asertividad) y +90 (máxima asertividad).

Se recolectó información de variables sociodemográficas de interés (edad, género, semestre actual, estrato socio-económico, número de se- mestres por salir graduado de la universidad y si la carrera de Enfermería o Fisioterapia había sido su primera elección al ingresar a la universidad).

\section{Análisis}

Todos los registros fueron sometidos a doble digitación y validados en Epi Info v. 6.04d. ${ }^{12}$ Se realizó un análisis descriptivo de las variables sociodemográficas, se aplicó la técnica del Análisis de Componentes Principales $\left(\mathrm{ACP}^{*}\right)$ y el Análisis de Factores (AF) a todos los 30 reactivos del RAS para conocer el aporte de éstos a la explicación y estructura de varianzas-covarianzas. ${ }^{13}$ Se hizo la matriz de correlación, se extrajeron los factores componentes principales, se graficó la pendiente de Cattell ${ }^{*}$, se obtuvo la estructura de los tres factores ponderados, se calculó el valor crítico y se realizó la rotación varimax. ${ }^{14}$ Para el $\mathrm{AF}$, el valor crítico se calculó en $0.35 .^{14}$

Finalmente se realizó un modelo de regresión lineal múltiple con los factores obtenidos; la manipulación de variables y los cálculos estadísticos, fueron realizados con STATA/SE v. 8.2. ${ }^{15}$

\section{Consideraciones éticas}

El proyecto de investigación fue aprobado por el Comité de Ética para la Investigación Científica de la Facultad de Salud de la Universidad Industrial de Santander. Los estudiantes participantes firmaron consentimiento informado y la recolección de la información se realizó por medio de encuesta auto-diligenciada garantizándose la confidencialidad de la información suministrada.

\section{RESULTADOS}

\section{Características de los participantes}

De los 225 estudiantes, 216 respondieron completamente el instrumento, lo que supone una respuesta del 96\%. La distribución de éstos fue: $174(77 \%)$ fueron estudiantes de Enfermería, $51(23 \%)$ fueron estudiantes de Fisioterapia. Con respecto al género, 173 (77\%) fueron mujeres y $52(23 \%)$ fueron hombres. La edad sigue una distribución normal con una media de 21 años y una desviación estándar de 2.87. El estrato socio-

\footnotetext{
* Técnica utilizada para reducir la dimensionalidad de un conjunto de datos. Los nuevos componentes principales o factores serán una combinación lineal de las variables originales, y además serán independientes entre sí.

** Representación gráfica de los autovalores de los factores.
} 
económico presenta una distribución normal con una media de 3 y una desviación estandar de 0.96. La distribución de los estudiantes por semestre se observa en la tabla 1.

Tabla 1 - Distribución por semestre académico en curso de los estudiantes de enfermería y fisioterapia de la Universidad Industrial de Santander. Bucaramanga-Colombia, 2003

\begin{tabular}{ccccc}
\hline $\begin{array}{c}\text { Semestre } \\
\text { académico }\end{array}$ & \multicolumn{2}{c}{$\begin{array}{c}\text { Enfermería } \\
(\mathbf{n}=174)\end{array}$} & \multicolumn{2}{c}{$\begin{array}{c}\text { Fisioterapia } \\
(\mathbf{n}=51)\end{array}$} \\
\hline 1 & $\mathbf{n}$ & $\%$ & $\mathbf{n}$ & $\%$ \\
3 & 22 & 12.64 & - & - \\
4 & - & - & 31 & 60.78 \\
5 & 27 & 5.52 & - & - \\
7 & 49 & 28.16 & - & - \\
8 & - & - & 20 & 39.22 \\
9 & 32 & 18.39 & - & - \\
\hline
\end{tabular}

Fuente: Base de datos STATA
Para 148 de los estudiantes (66\%) su carrera, Enfermería o Fisioterapia, según corresponda, fue su primera elección al ingresar a la universidad, mientras para 76 de los estudiantes (34\%), no lo fue.

En cuanto al puntaje de asertividad del RAS en la población estudiada, se encontró una media de 8.8 y una desviación estandar de 20.9 con un puntaje mínimo de -45 y máximo de 69 . En relación al ACP y AF se obtuvo que de los 30 reactivos del RAS se extrajeron 3 factores componentes principales con los cuales se explica el 73\% de la varianza y se denominaron: F1 (Asertividad indirecta: persona asertiva pero sólo si no tiene que enfrentarse cara a cara con la persona o situación de conflicto), F2 (No asertividad: persona no asertiva o socialmente inhábil para expresar sus deseos, opiniones, sentimientos, etc.) y F3 (Asertividad: persona asertiva o socialmente hábil para expresar sus limitaciones, sentimientos, opiniones, etc.).

Se graficó la pendiente de Cattell (Figura 1) y se realizó la rotación varimax ${ }^{* \star *}$ de los factores (Tabla 2). Las medidas de tendencia central y de dispersión de los factores obtenidos se pueden observar en la tabla 3.

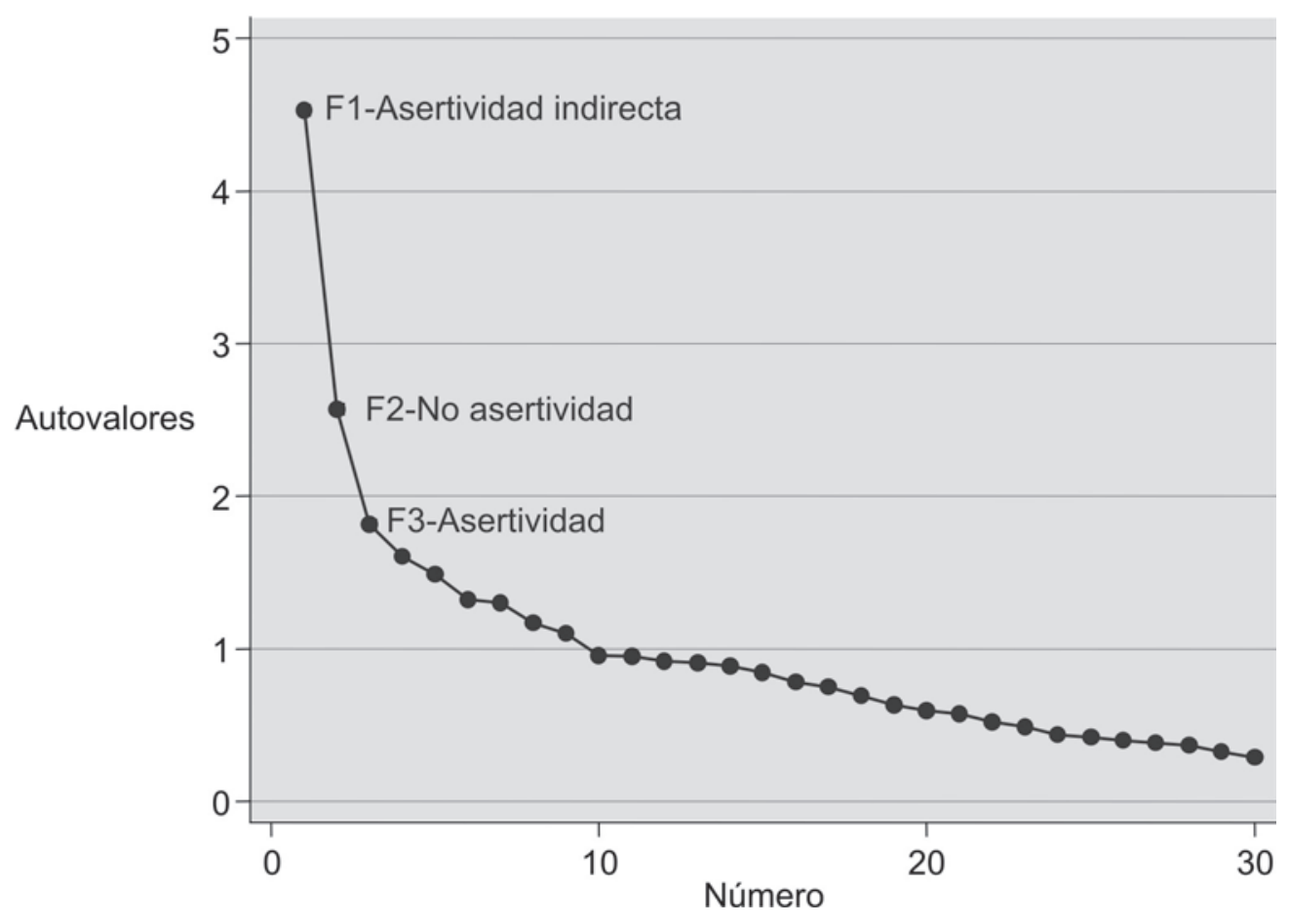

Figura 1 - Pendiente de Cattell. Bucaramanga-Colombia, 2003

*** El método varimax maximiza la suma de varianzas de las cargas requeridas de la matriz de factores. 
Tabla 2 - Autovalores con rotación varimax de los factores en los estudiantes de enfermería y fisioterapia de la Universidad Industrial de Santander. Bucaramanga-Colombia, 2003

\begin{tabular}{|c|c|c|c|}
\hline Variable & $\begin{array}{c}\text { Factor } 1 \\
\text { Asertividad indirecta }\end{array}$ & $\begin{array}{c}\text { Factor } 2 \\
\text { No asertividad }\end{array}$ & $\begin{array}{c}\text { Factor } 3 \\
\text { Asertividad }\end{array}$ \\
\hline Reactivo 1 & 0.07821 & 0.19189 & -0.02317 \\
\hline Reactivo 2 & 0.29886 & 0.25382 & 0.15716 \\
\hline Reactivo 3 & -0.12725 & -0.00496 & -0.37552 \\
\hline Reactivo 4 & 0.00619 & 0.16659 & 0.11310 \\
\hline Reactivo 5 & 0.08356 & 0.50896 & 0.03459 \\
\hline Reactivo 6 & -0.32058 & -0.01975 & -0.14554 \\
\hline Reactivo 7 & -0.17911 & 0.06147 & -0.40907 \\
\hline Reactivo 8 & -0.29946 & -0.01593 & -0.31467 \\
\hline Reactivo 9 & 0.17586 & 0.44857 & -0.06196 \\
\hline Reactivo 10 & -0.36327 & 0.14843 & -0.06400 \\
\hline Reactivo 11 & 0.21749 & 0.46614 & 0.16690 \\
\hline Reactivo 12 & 0.44197 & 0.27329 & -0.01795 \\
\hline Reactivo 13 & 0.55714 & 0.25450 & -0.02924 \\
\hline Reactivo 14 & 0.11754 & 0.44409 & 0.16463 \\
\hline Reactivo 15 & 0.10614 & 0.26413 & 0.28063 \\
\hline Reactivo 16 & 0.53101 & 0.33549 & 0.00054 \\
\hline Reactivo 17 & 0.08182 & 0.21669 & -0.10539 \\
\hline Reactivo 18 & -0.43461 & 0.04682 & -0.07298 \\
\hline Reactivo 19 & 0.09891 & 0.27854 & 0.08243 \\
\hline Reactivo 20 & -0.13058 & 0.12699 & -0.23972 \\
\hline Reactivo 21 & -0.51023 & 0.05442 & -0.10741 \\
\hline Reactivo 22 & -0.13167 & 0.01745 & -0.41417 \\
\hline Reactivo 23 & -0.07459 & 0.66208 & -0.05112 \\
\hline Reactivo 24 & 0.22202 & 0.37711 & 0.00166 \\
\hline Reactivo 25 & -0.31178 & -0.04509 & -0.36760 \\
\hline Reactivo 26 & 0.13607 & 0.36995 & 0.15001 \\
\hline Reactivo 27 & -0.00485 & 0.00730 & -0.64531 \\
\hline Reactivo 28 & -0.03176 & -0.12393 & -0.51437 \\
\hline Reactivo 29 & -0.68867 & 0.03846 & -0.14184 \\
\hline Reactivo 30 & 0.42374 & 0.39408 & -0.04960 \\
\hline
\end{tabular}

Fuente: Base de datos STATA.

Tabla 3 - Parámetros estadísticos de los factores en los estudiantes de enfermería y fisioterapia de la Universidad Industrial de Santander. Bucaramanga-Colombia, 2003

\begin{tabular}{lcccc}
\hline Factores & Mediana & Desviación estándar & Valor mínimo & Valor máximo \\
\hline F1-Asertividad indirecta & 0.02 & 0.88 & -2.40 & 1.79 \\
F2-No aserti-vidad & 0.09 & 0.87 & -2.06 & 1.99 \\
F3-Asertividad & 0.04 & 0.84 & -2.41 & 1.81 \\
\hline
\end{tabular}

Fuente: Base de datos STATA.

En la tabla 4, se pueden apreciar los parámetros estadísticos de la variable asertividad en la población de estudio. Se aprecia que el puntaje de asertividad fue mayor en los estudiantes de fisioterapia. 
Tabla 4 - Parámetros estadísticos del puntaje de asertividad en los estudiantes de enfermería y fisioterapia de la Universidad Industrial de Santander. Bucaramanga-Colombia, 2003

\begin{tabular}{lcccccc}
\hline Estudiantes & $\begin{array}{c}\text { Puntaje promedio } \\
\text { de asertividad }\end{array}$ & Valor mínimo & Valor máximo & Percentil 25\% & Mediana & Percentil 75\% \\
\hline Enfermería & 7.41 & -45 & 69 & -4 & 7 & 21 \\
Fisioterapia & 13.64 & -32 & 64 & 3 & 13 & 22 \\
\hline
\end{tabular}

Fuente: Base de datos STATA.

Se determinó que de los 30 reactivos que conforman el RAS, nueve reactivos presentan valores menores que el valor crítico y por lo tanto no pesaron en ningún factor; también se observó que el reactivo 30 pesó en dos factores (Tabla 2). Finalmente se obtuvo que el factor 1 (Asertividad indirecta) agrupó ocho reactivos, el factor 2 (No asertividad) agrupó ocho reactivos y el factor 3 (Asertividad) agrupó seis reactivos (Tabla 2).

En la tabla 5, se puede apreciar que a medida que aumenta la edad, aumenta la asertividad indirecta (factor 1) por cada año en 0.04 y se ajusta, si se es hombre. También la edad es importante en el factor 2; en la tabla 6, se muestra que a medida que aumenta la edad, disminuye la no asertividad (factor $2)$, siendo estadísticamente significativo $(\mathrm{p}=0.004)$.

Igualmente, se encontró que entre más semestres por salir graduado de la universidad, es menor la Asertividad (factor 3 ) en -0.05 , siendo estadísticamente significativo $(\mathrm{p}=0.005)$; la tabla 7 , resume estos hallazgos.

Tabla 5 - Factor (1) Asertividad indirecta. Estudiantes de enfermería y fisioterapia de la Universidad Industrial de Santander. BucaramangaColombia, 2003

\begin{tabular}{lcccc}
\hline Variable & Coeficiente & Valor $(\mathbf{p})$ & IC (95\%) \\
\hline Edad & 0.04 & 0.041 & 0.00 & 0.08 \\
Género Masculino & 0.21 & 0.150 & -0.06 & 0.49 \\
Intercepto & -0.97 & 0.033 & -1.87 & -0.08 \\
\hline
\end{tabular}

IC (95\%): Intervalo de confianza del 95\%.

Fuente: Base de datos STATA.

Tabla 6 - Factor (2) No asertividad. Estudiantes de enfermería y fisioterapia de la Universidad Industrial de Santander. Bucaramanga-Colombia, 2003

\begin{tabular}{lcccc}
\hline Variable & Coeficiente & Valor (p) & \multicolumn{2}{c}{ IC (95\%) } \\
\hline Edad & 0.06 & 0.004 & 0.01 & 0.10 \\
Intercepto & -1.30 & 0.004 & -2.17 & -0.40 \\
\hline
\end{tabular}

IC (95\%): Intervalo de confianza del 95\%.

Fuente: Base de datos STATA .
Tabla 7 - Factor (3) Asertividad. Estudiantes de enfermería y fisioterapia de la Universidad Industrial de Santander. Bucaramanga-Colombia, 2003

\begin{tabular}{|c|c|c|c|c|}
\hline Variable & Coeficiente & Valor (p) & \multicolumn{2}{|c|}{ IC (95\%) } \\
\hline $\begin{array}{l}\text { Semestre por } \\
\text { salir graduado de } \\
\text { la Universidad }\end{array}$ & -0.05 & 0.005 & -0.09 & -0.01 \\
\hline Intercepto & 0.24 & 0.019 & 0.04 & 0.44 \\
\hline
\end{tabular}

IC (95\%): Intervalo de confianza del 95\%.

Fuente: Base de datos STATA.

\section{DISCUSIÓN}

El presente estudio pone de manifiesto que la edad, es un elemento fundamental, para que las personas sean asertivas; a medida que aumenta la edad disminuye el riesgo de no ser asertivos; lo cual es similar a los resultados obtenidos en 1998 por un autor en su estudio de asertividad de los profesionales de enfermería en situaciones conflictivas, donde destacan que las enfermeras de mayor edad muestran una actitud más asertiva. ${ }^{8}$

En cuanto a las limitaciones del estudio debemos hacer constar como posible distorsionante, la deseabilidad social, entendida ésta como el deseo del encuestado de agradar a los investigadores, (puesto que en algunas ocasiones los estudiantes pensaban que era un trabajo para valorar sus capacidades); lo cual podría influir en la respuesta a las preguntas del instrumento y se podía constituir en un sesgo que se intentó minimizar garantizando el anonimato.

De otra parte, se podrían comparar los resultados de éste estudio, en cuanto a la asertividad indirecta, con los hallazgos obtenidos en 1998, donde encontraron profesionales asertivos que en algunas situaciones eran capaces de expresar sus opiniones y sentimientos, pero no eran hábiles para asumir las repercusiones o beneficios que pueden derivarse de una postura de oposición. ${ }^{9}$

Se evidencia también en el presente estudio, que entre más semestres por salir graduado de la universidad, es menor la asertividad; sería 
interesante repetir el estudio, tras un período de formación específica en el área de la asertividad, pues si se empieza un entrenamiento asertivo desde los primeros semestres, se podría valorar el posible incremento de ésta. ${ }^{16}$

\section{CONCLUSIONES}

El comportamiento asertivo se centra en negociar cambios razonables respecto a la manera como ambas partes se conducen en las relaciones interpersonales, a fin de lograr el equilibrio en la interacción social. Es por ello que debe incrementarse la formación en el área de la asertividad y las habilidades sociales con el objetivo de disminuir las dificultades y conflictos en las relaciones interpersonales.

Estos resultados respaldan la necesidad de implementar estrategias para que la asertividad como habilidad relacional sea contemplada como requisito en la formación de los profesionales de la salud, ya que aplicada correctamente permite una mayor y más completa consecución de los cuidados y servicios que se prestan y por lo tanto un mayor reconocimiento profesional y personal.

\section{REFERENCIAS}

1. Flores MM, Díaz R. Escala multidimensional de asertividad. México: Manual Moderno; 2003.

2. Caballo, VE. Manual de evaluación y entrenamiento de las habilidades sociales. $7^{\text {a }}$ edición Madrid (ES): Siglo XXI España Editores; 2007.

3. Salter A. Conditioned Reflex Therapy: the direct approach to the reconstruccion of personality. New York (US): Creative Age Press; 1949.
4. Wolpe J. Psychotherapy by reciprocal inhibition. Palo Alto (US): Standford University Press; 1958.

5. Lazarus AA. Behavior rehearsal vs. non-directive therapy vs. advine in affecting behaviour change. Behav Res Ther. 1966 Aug; 4(3):209-12.

6. Davidson J. Asertividad. México: Prentice Hall; 1999.

7. Castanyer O. La asertividad: expresión de una sana autoestima. 31 a edición Bilbao (ES): Desclée de Brower; 2010.

8. Hernández AM, Mirabent J, Sariol T, Boix E. Asertividad de los profesionales de enfermería frente a situaciones conflictivas de tipo relacional con el enfermo y/o los familiares. Enferm Clín, 1997; 7(5):212-6.

9. Cabrera S, Guil R, Lax P. Asertividad en enfermería: ¿una característica de la profesión? Enferm Clin. 1998; 8(3):98-103.

10. Hernández B, Velasco HE. Encuestas transversales. Salud Pública Méx. 2000 Sep; 42(5):447-55.

11. Rathus S. A 30-item schedule for assessing assertive behavior. Behav Ther. 1973 May; 4:398-406.

12. CDC de Atlanta. Epi Info (programa de ordenador) versión 6.04d. Atlanta (US): Epidemiología en ordenadores; 2001.

13. Godshalk EB, Timothy DH. Factor and principal component analyses as alternatives to index selection. Teor Apll Genet. 1998; 76:352-360.

14. Streiner DL. Bioestadística. Madrid (ES): Mosby/ Doyma; 1996 p.129-41.

15. StataCorp. Stata statistical software: Release 8.2 College Station, TX: Stata Corporation; 2003.

16. Mc Cabe C, Timmins F. Teaching assertiveness to undergraduate nursing students. Nurse Educ Pract. 2003 Mar; 3(1):30-42. 